\title{
Familial Mediterranean Fever in Algeria - A Retrospective of Three Molecular Studies
}

\section{Type of article: conference abstract}

\author{
Djouher Ait-Idir ${ }^{1}$, Bahia Djerdjouri ${ }^{2}$
}

${ }^{1}$ Department of Biology, Faculty of Sciences, M'Hamed Bougara University, Boumerdes, Algeria

${ }^{2}$ Faculty of Biological Sciences, University of Sciences and Technology Houari Boumediene, Bab-Ezzouar, Algeria

Corresponding Author: d.aitidir@univ-boumerdes.dz

\begin{abstract}
:
Background: Familial Mediterranean fever (FMF, OMIM 249100) is an autosomal recessive disease characterized by episodic febrile attacks and polyserositis. Renal AA-amyloidosis can complicate FMF. MEFV is the gene responsible for FMF and is involved in the regulation of inflammation. Although FMF is endemic in the Mediterranean region, its diagnosis is very recent in Algeria. We present here a retrospective of three genetic studies carried out on the Algerian patients.

Methods: 183 unrelated patients with symptoms suggestive of FMF were recruited from various hospitals between 2007 and 2015. Molecular studies included three cohorts of patients: 71 ( 35 males, 36 females), 84 (42 males, 42 females), and 28 (15 males, 13 females) with renal AA-amyloidosis. We searched for mutations in exon 10 of the $M E F V$ gene by allele-specific PCR (p.M694V, p.M694I, p.M680I and p.A744S) and by resequencing the entire coding region of the same exon after PCR amplification.

Results: Molecular analysis identified 152 mutant alleles among 94/183 patients (51.36\%). p.M694I was the most predominant mutation accounting for $63.2 \%$ of mutated alleles, followed by p.M694V (15.13\%), p.M680I (13.81\%), p.I692del $(1.32 \%)$ and p.K695R (0.66\%). More importantly, the M694I/M694I genotype was the most prevalent among the patients with AA-amyloidosis than the M694V/M694V genotype.
\end{abstract}

Conclusion: Our results provide the first genetic data concerning FMF in Algeria. They show that p.M694I mutation could be responsible for the severe phenotype for Algerian FMF patients.

Keywords: AA-Amyloidosis, Algeria, Familial Mediterranean Fever, P.M694I Mutation.

\section{Declaration of conflicts}

This article is a conference abstract selected from the abstract book of the International Congress on Health Sciences and Medical Technologies ICHSMT'19, Tlemcen, Algeria, December 05-07, 2019. 
Medical Technologies Journal, Volume: 3, Issue: 4, October-December 2019, Pages: 487-488. Doi :

https://doi.org/10.26415/2572-004X-vol3iss4p487-488

\section{Authors' Biography}

No biography

\section{References}

No references 